Bryn Mawr College

Scholarship, Research, and Creative Work at Bryn Mawr

College

Physics Faculty Research and Scholarship

Physics

2016

\title{
Methyl and t-butyl group rotation in a molecular solid: 1 H NMR spin-lattice relaxation and X-ray diffraction
}

Peter A. Beckmann

Bryn Mawr College, pbeckman@brynmawr.edu

Curtis E. Moore

Arnold L. Rheingold

Let us know how access to this document benefits you.

Follow this and additional works at: http://repository.brynmawr.edu/physics_pubs

Part of the Physics Commons

\section{Custom Citation}

Methyl and t-butyl group rotation in a molecular solid: $1 \mathrm{H}$ NMR spin-lattice relaxation and X-ray diffraction. P A Beckmann, C E Moore, and A L Rheingold 2016 Physical Chemistry Chemical Physics, 18 1720-1726.

This paper is posted at Scholarship, Research, and Creative Work at Bryn Mawr College. http://repository.brynmawr.edu/physics_pubs/91

For more information, please contact repository@brynmawr.edu. 


\title{
Methyl and $t$-butyl group rotation in a molecular solid: ${ }^{1} \mathrm{H}$ NMR spin-lattice relaxation and $\mathrm{X}$-ray diffraction
}

\author{
Peter A. Beckmann, ${ }^{a^{*}}$ Curtis E. Moore ${ }^{\mathrm{b}}$, and Arnold L. Rheingold ${ }^{\mathrm{b}}$
}

\begin{abstract}
a Department of Physics, Bryn Mawr College, Bryn Mawr, Pennsylvania 19010-2899, USA. Email:pbeckman@brynmawr.edu

${ }^{b}$ Department of Chemistry and Biochemistry, University of California, San Diego, 9500 Gilman Dr., La Jolla, California 92093-0358, USA. Email: arheingold@ucsd.edu
\end{abstract}

2016 Physical Chemistry Chemical Physics, 18 1720-1726.

\section{Abstract}

We report solid state ${ }^{1} \mathrm{H}$ nuclear magnetic resonance spin-lattice relaxation experiments and X-ray diffractometry in 2-t-butyldimethylsilyloxy-6-bromonaphthalene. This compound offers an opportunity to simultaneously investigate, and differentiate between, the rotations of a $t$-butyl group $\left[\mathrm{C}\left(\mathrm{CH}_{3}\right)_{3}\right]$ and its three constituent methyl groups $\left(\mathrm{CH}_{3}\right)$ and, simultaneously, a pair of 'lone' methyl groups (attached to the Si atom). The solid state ${ }^{1} \mathrm{H}$ relaxation experiments determine activation energies for these rotations. We review the models for the dynamics of both 'lone' methyl groups (ones whose rotation axes do not move on the NMR time scale) and models for the dynamics of the $t$-butyl group and its constituent methyl groups (whose rotation axes reorient on the NMR time scale as the $t$-butyl group rotates).

\section{Introduction}

Solid state nuclear magnetic resonance (NMR) relaxation experiments ${ }^{1}$ can be used to develop robust models for the dynamics of intramolecular groups like $t$-butyl groups $\left[\mathrm{C}\left(\mathrm{CH}_{3}\right)_{3}\right]$ and their constituent methyl groups $\left(\mathrm{CH}_{3}\right)^{2}$ or, just methyl groups bonded to a rigid backbone. ${ }^{3}$ These relaxation experiments measure the NMR frequency Fourier component of changing magnetic fields ${ }^{1}$ and this information can be related to motion occurring on the NMR time scale $\left(10^{-10}\right.$ to $10^{-}$ ${ }^{5} \mathrm{~s}$ for the experiments reported here). This technique is not very good unless one knows a priori that there are very few motions on the NMR time scale, and even then, models must be employed. 
Fortunately, molecular vibrations and librations (bond lengths, angles, lattice vibrations, etc.) occur on a much faster time scale (typically $10^{-15}-10^{-14} \mathrm{~s}$ ). These methyl group and $t$-butyl group rotations are characterized by barriers in the $5-30 \mathrm{~kJ} \mathrm{~mol}^{-1}\left(1-7 \mathrm{kcal} \mathrm{mol}^{-1}\right)$ range and very few experimental techniques can study dynamical processes at these low energies. The compound studied here is 2-t-butyldimethylsilyloxy-6-bromonaphthalene (Fig. 1). We report both the crystal structure from an X-ray diffraction experiment and the temperature dependence of the various parameters that characterize the somewhat complicated nonexponential solid state NMR ${ }^{1} \mathrm{H}$ spinlattice relaxation in a polycrystalline sample. The asymmetric unit ${ }^{4}$ in the crystal is a single molecule so that makes for a clean assignment of rotational barriers since all molecules are equivalent. The molecule chosen for this study is representative of a large class of compounds that have a variety of rotors with quite different dynamics and as such is a test of the models for methyl and $t$-butyl group rotation. ${ }^{2,3,5,6}$ The novelty of this work is that different sets of neighbouring methyl groups (those attached to the $\mathrm{Si}$ atom and those in the $t$-butyl group) in a relatively complicated molecule in a solid sample have been identified and characterized by their dynamical properties. Readers not interested in the details of the X-ray and solid state NMR relaxation experiments are invited to go directly to the Conclusions.

\section{Experiments}

\subsection{Single crystal X-ray diffraction}

The sample of 2-t-butyldimethylsilyloxy-6-bromonaphthalene was purchased from Sigma Aldrich and used as is. The quoted purity was $97 \%$. A single crystal, taken from the same sample used to perform the NMR relaxation experiments, was mounted on a Hampton CryoLoop with Paratone-N and data collected with a Bruker D8 diffractometer using an Ultra rotating-anode generator (Mo) equipped with a high-efficiency multi-layer, double-bounce monochromator. Experimental details are collected in Table 1. All data were collected with $1.0 \mathrm{sec} / 1.0^{\mathrm{O}}$ correlated scans. Structure solution and subsequent refinement used various components of the SHELXTL software package distributed by the Bruker Corporation (G. Sheldrick, Bruker-AXS, Madison, Wisconsin, USA). The geometry of the molecule in the crystal is shown in Fig. 1 and the crystal structure (the 100 and 101 planes) is shown in Fig. 2. 


\subsection{Powder X-ray diffraction}

A powder X-ray diffractogram was taken with the same sample used for the NMR spin-lattice relaxation experiments. The sample was ground to a thick paste in mineral oil and spread evenly on a fine Nylon loop. Data were recorded using a Cu rotating-anode source and a Vantac 500 detector at 100K. As indicated in Fig. 3, the powder diffractogram and the simulated powder diffractogram calculated from the single-crystal data, compare very closely, indicating that the bulk sample does not contain any significant impurities.

\subsection{NMR spin-lattice relaxation}

The solid state NMR ${ }^{1} \mathrm{H}$ spin-lattice relaxation experiments were performed with a polycrystalline sample between 88 and $312 \mathrm{~K}$ at NMR frequencies of $\omega / 2 \pi=53.0,22.5$, and 8.50 $\mathrm{MHz}$ (in applied magnetic fields of 1.24, 0.528, and $0.200 \mathrm{~T}$ ). The NMR frequencies being used in these relaxation experiments are very low compared with conventional high resolution NMR spectroscopy experiments. This is needed in order to bring the frequencies of the intramolecular motions being studied into resonance with the NMR frequencies in a temperature range below the melting points of the solids like that being studied here. Signal-to-noise at the lowest NMR frequency $(8.50 \mathrm{MHz})$ was insufficient to make measurements below approximately $110 \mathrm{~K}$ mainly because the narrowing free induction decay, characterized by a spin-spin relaxation time of approximately $5 \mu \mathrm{s}$, became lost in the amplifier recovery time in the pulsed NMR experiment. The approximate spin-spin relaxation time of $5 \mu$ s corresponds to a line width of approximately $200 \mathrm{kHz}$ or $23,000 \mathrm{ppm}$ (at $8.50 \mathrm{MHz}$ ). A (perturbation $\pi$ pulse)-t-(observe $\pi / 2$ pulse) sequence was used and the return of the perturbed magnetization to its equilibrium value $M(\infty)$ was monitored. The time dependence of the ${ }^{1} \mathrm{H}$ magnetization $M(t)$ was always fitted to a single exponential $M(t)=M(\infty)[1-(1-\cos \theta)\{\exp (-R t)\}]$ and to a stretched exponential $M(t)=$ $M(\infty)\left[1-(1-\cos \theta)\left\{\exp \left(-R^{*} t\right)^{\beta}\right\}\right]^{7-16}$ A minireview of the use of the stretched exponential function

in the physical sciences is provided elsewhere. ${ }^{9} R$ is the unique spin-lattice relaxation rate (inverse of the spin-lattice relaxation time $T_{1}$ ), $R^{*}$ is the characteristic relaxation rate, $\beta$ is the stretching parameter, and $\theta$ is a parameter that accounts for the imperfections in the perturbing $\pi$ pulse.

When $\beta>0.95, R$ for a single exponential and $R^{*}$ for a stretched exponential are within a few percent of each other and the relaxation is deemed "exponential." The temperature 
dependence of $\beta$ is shown in Fig. 4 where $\beta=0.95$ is indicated by the dashed horizontal line. Note that there is considerable scatter in these data and that $\beta$ is essentially independent of NMR frequency. The uncertainties are large at high temperature and smaller at low temperature. Indeed, the large uncertainties at high temperature plus the fact that $\beta>0.95$ essentially tell us that the relaxation can be taken to be exponential and a stretched exponential fit is really not necessary. But this must be determined. At the same time, $\beta \approx 0.87$ at low temperatures and the relaxation cannot be taken to be exponential. That is, the relaxation was deemed nonexponential for temperatures below approximately 140,130 , and $110 \mathrm{~K}$ at $53.0,22.5$, and $8.50 \mathrm{MHz}$ (Fig. 4). It was deemed exponential above these temperatures (i.e., $\beta>0.95$ ). When the relaxation was nonexponential, the initial (short time) slope of the perturbed magnetization was determined (as outlined elsewhere ${ }^{9}$ ) and this parameter, discussed further in the Results section, is called $R_{\mathrm{S}}$.

The temperature dependence of the relaxation rates $R, R_{\mathrm{S}}$, and $R^{*}$ is shown in Fig. 5. Here, the same symbols are used for $R$ and $R_{\mathrm{S}}$ (which are fitted to the same model in the Results section) and different symbols are used for $R^{*}$ (which are not amenable to theoretical interpretation). The transition from exponential to nonexponential relaxation can be seen at the same temperatures in Figs. 4 and 5. The uncertainties indicated for $R$ and $R^{*}$ are larger than the values returned by standard nonlinear fitting routines. More meaningful uncertainties were determined in a separate numerical exercise by adding random noise to noiseless relaxation decays (to mimic the real experiments) and performing a statistical study (using approximately 2000 simulations in each case) of the resulting rates. As a result, the uncertainties on $R$ and $R^{*}$ at 22.5 and $53.0 \mathrm{MHz}$ are taken to be $\pm 7 \%$, the uncertainties on $R$ and $R^{*}$ at $8.50 \mathrm{MHz}$ are taken to be $\pm 10 \%$, and the uncertainties on $R_{\mathrm{S}}$ at all three frequencies are taken to be $\pm 20 \%$. The size of the solid symbols for $R$ (high temperatures) and $R^{*}$ (low temperatures) at 53.0 and $22.5 \mathrm{MHz}$ in Fig. 5 are chosen to reflect, approximately, the uncertainties while the uncertainties for $R$ at $8.50 \mathrm{MHz}$ and for $R_{\mathrm{S}}$ at all three frequencies are visible in Fig. 5.

Temperature was controlled with a cold nitrogen gas flow system and temperatures were measured with home-made, silver-soldered, copper-constantan thermocouples that are carefully calibrated with four secondary temperature standards every few years. The polycrystalline sample of 2-t-butyldimethylsilyloxy-6-bromonaphthalene was in a $6 \mathrm{~mm}$ ID tube that contained $20 \mathrm{~mm}$ of sample. The NMR coil was $15 \mathrm{~mm}$ in length meaning that $5 \mathrm{~mm}$ of sample was outside the coil. The thermocouple was imbedded in this additional $5 \mathrm{~mm}$ of sample. Two samples were used with 
several thermocouples at two completely independent experimental stations to make sure there were no systematic effects related to apparatus, temperature control, or slightly different measurement procedures.

\section{Results}

The ${ }^{1} \mathrm{H}$ spin-lattice relaxation results from the modulation of the ${ }^{1} \mathrm{H}-{ }^{1} \mathrm{H}$ spin-spin interactions by methyl group and $t$-butyl group rotation. All other motions take place on time scales too far removed from the NMR time scale (characterized by being within approximately two orders of magnitude of the inverse NMR frequencies) to be effective at relaxing the ${ }^{1} \mathrm{H}$ spin system. The spin-lattice relaxation for an ensemble of randomly oriented and isolated methyl $\left(\mathrm{CH}_{3}\right)$ groups (which involves the modulation of only intraCH${ }_{3}{ }^{1} \mathrm{H}-{ }^{1} \mathrm{H}$ interactions) is inherently nonexponential because of the anisotropic nature of the rotation (the spin-spin vectors orient in a plane) and because the motion of the three spin-spin vectors are perfectly correlated. ${ }^{17,}{ }^{18} \mathrm{CH}_{3}$-nonCH${ }_{3}{ }^{1} \mathrm{H}-{ }^{1} \mathrm{H}$ interactions and interCH${ }_{3}{ }^{1} \mathrm{H}-{ }^{1} \mathrm{H}$ interactions tend to make the relaxation more exponential. ${ }^{19,20}$ When the $\mathrm{CH}_{3}$ rotation axis is moving on the NMR time scale (as is the case for a $t$-butyl group), the relaxation, also, tends to be more exponential. ${ }^{21,22}$ Indeed, these observations are to be found in the two main messages from the somewhat messy looking Fig. 4. First, $\beta$, the parameter that characterizes the degree of nonexponential relaxation, is independent of NMR frequency (within experimental uncertainty) except in the transition region between the low and high temperature parts of Fig. 4. Second, $\beta>0.95$ at high temperatures where the rotation of the $t$-butyl groups and their constituent methyl groups dominate and $\beta=0.87 \pm 0.02$ at low temperatures where the rotation of the methyl groups attached to the silicon atom dominate the relaxation. This $\beta \approx 0.9$ is the standard signature for a methyl group rotating faster than the inverse NMR frequency. ${ }^{8}$ Here, $R_{\mathrm{S}}$ (the initial slope) and $R^{*}$ (in the stretched exponential) differ significantly (Fig. 5). In all cases, it is the initial relaxation rate $R_{\mathrm{S}}$ following a perturbation that is amenable to a theoretical model (next paragraph). This is because, at short times following the perturbation, the effects of spinspin correlations and anisotropic motion have not yet "stretched" the relaxation. ${ }^{17,} 18$ If $\beta>0.95, R$ $\approx R_{\mathrm{S}}$ and the same model can be applied to $R$.

We consider the case of thermally assisted classical rotation since quantum mechanical tunneling need not be considered above approximately $80 \mathrm{~K}^{23-30}$ Below approximately $80 \mathrm{~K}$, tunneling occurs and a different model must be used. ${ }^{31}$ We first consider just the methyl groups 
bonded to the Si atom. The rotation axes of these methyl groups are not moving on the NMR time scale. The initial relaxation rate $R_{\mathrm{S}}$ of a perturbed ${ }^{1} \mathrm{H}$ magnetization is ${ }^{9,32-34} R_{\mathrm{S}}=(n / N)\left(A_{\text {intra }}+\right.$ $\left.A_{\text {inter }}\right)[J(\omega, \tau)+4 J(2 \omega, \tau)]=(n / N)(1+y) A_{\text {intra }}[J(\omega, \tau)+4 J(2 \omega, \tau)]$ with $J(\omega, \tau)=2 \tau /\left(1+\omega^{2} \tau^{2}\right), \tau=$ $\tau_{0}\left[\exp \left(E_{\mathrm{NMR}} / k T\right)\right]$, and $y=A_{\text {inter }} / A_{\text {intra. }}$. The parameter $n$ (=6 for the two Si methyl group H atoms) is the number of ${ }^{1} \mathrm{H}$ spins involved with the motion and $N(=21)$ is the number of ${ }^{1} \mathrm{H}$ spins in the asymmetric unit which, in this case, is the whole molecule. The ratio $n / N$ can be thought of as a dilution factor. The relaxation is "slowed down" by $n / N$ as the $n$ "relaxing ${ }^{1} \mathrm{H}$ spins" relax the $N-$ $n$ "nonrelaxing ${ }^{1} \mathrm{H}$ spins" following a perturbation. This assumes that spin diffusion is effective. $A_{\text {intra }}$ is a constant that characterizes the $\operatorname{six}{ }^{1} \mathrm{H}_{-}{ }^{1} \mathrm{H}$ spin-spin interactions among the three ${ }^{1} \mathrm{H}$ spins in a $\mathrm{CH}_{3}$ group and can be calculated in terms of the $\mathrm{H}-\mathrm{H}$ distances and other constants. ${ }^{34}$ The parameter $A_{\text {inter }}$ characterizes the contribution to the relaxation of the $\mathrm{CH}_{3}-$ nonCH$_{3}{ }^{1} \mathrm{H}-{ }^{1} \mathrm{H}$ interactions and the interCH${ }_{3}{ }^{1} \mathrm{H}-{ }^{1} \mathrm{H}$ interactions. $A_{\text {inter }}$ would be very difficult to calculate since it involves many ${ }^{1} \mathrm{H}-{ }^{1} \mathrm{H}$ interactions with varying $\mathrm{H}-\mathrm{H}$ separations $r$ and many different angular variations of the vectors $\vec{r}$ (as the methyl group rotates). However, it is much smaller that $A_{\text {intra. }}$ It is convenient to use the dimensionless parameter $y=A_{\text {inter }} / A_{\text {intra }}$ which characterizes the ratio of the $\mathrm{CH}_{3}-$ nonCH ${ }_{3}{ }^{1} \mathrm{H}-{ }^{1} \mathrm{H}$ interactions and the interCH${ }_{3}{ }^{1} \mathrm{H}-{ }^{1} \mathrm{H}$ interactions to the intraCH${ }_{3}{ }^{1} \mathrm{H}-{ }^{1} \mathrm{H}$ interactions. As such, $A_{\text {intra }}+A_{\text {inter }}=A_{\text {intra }}(1+y)$. The product $A_{\text {intra }}(1+y)$ in reference 34 (where additional details are provided) is just called $A$ and was taken as a fitting parameter in that earlier work. Here, $A$ is replaced by $A_{\text {intra }}(1+y), A_{\text {intra }}$ is calculated, and $y$ (typically between 0 and 0.2$)$ is taken as the fitting parameter. $J(\omega, \tau)$ is the spectral density (the frequency spectrum of the local magnetic field resulting from methyl group rotation), $\tau$ can be taken as the mean time between methyl group hops, ${ }^{23-30,32,33} \tau_{0}$ is the preexponential factor, ${ }^{23,35,36}$ and $E_{\mathrm{NMR}}$ is the NMR activation energy. The preexponential factor $\tau_{0}$ can be modeled, crudely, by assuming the methyl group librates as a harmonic oscillator at the bottom of the well. In this case, ${ }^{37} \tau_{0}$ is $\tau_{\mathrm{HO}}=$ $[2 \pi / 3]\left[I /\left(2 E_{\mathrm{NMR}}\right)\right]^{1 / 2}$, where $I$ is the moment of inertia of a methyl group.

The adjustable parameters in the single fit to the temperature dependence of the relaxation rate $R_{\mathrm{S}}$ at low temperature at both 53.0 and $22.5 \mathrm{MHz}$ are $E_{\mathrm{NMR}}, y$, and $\tau_{0} / \tau_{\mathrm{HO}}$. It is clear from the environments of the two Si methyl groups (Fig. 2) that the two groups are not strictly equivalent. As a result, these three adjustable parameters will likely be slightly different for the two groups. However, the precision of the fits (next paragraph) are not sufficiently sensitive to these small differences and we treat the two methyl groups as dynamically equivalent. 
Since only the high-temperature, fast-motion limit of the rotation of the two methyl groups attached to the Si atom is observed $\left[\omega \tau<<1\right.$ in $J(\omega, \tau)=2 \tau /\left(1+\omega^{2} \tau^{2}\right)$ so $\left.J(\omega, \tau)=2 \tau\right]$ at low temperatures in Fig. 5, $R_{\mathrm{S}}=(n / N)(1+y) A_{\text {intra }}[10 \tau]=10(n / N)(1+y) A_{\text {intra }} \tau_{0}\left[\exp \left(E_{\mathrm{NMR}} / k T\right)\right]$ and only $E_{\mathrm{NMR}}$ (obtained from the slope of $\ln R_{\mathrm{S}}$ versus $T^{1}$ ) and the product $(1+y) A_{\text {intra }} \tau_{0}$ (obtained from the intercept of $\ln R_{\mathrm{S}}$ versus $T^{-1}$ ) can be determined. The fit of the data gives $E_{\mathrm{NMR}}=5 \pm 1 \mathrm{~kJ} \mathrm{~mol}^{-1}$. The two Si methyl groups might very well have different values of $E_{\mathrm{NMR}}$ within this $20 \%$ uncertainty. When $A_{\text {intra }}$ is set to its calculated value then $(1+y) \tau_{0} / \tau_{\mathrm{HO}}=3.0 \pm 1.5$. The value of $y$ is typically between 0 and 0.2 so $\tau_{0} / \tau_{\mathrm{HO}}$ is in the range 1-5. Again, the two Si methyl groups will likely have slightly different values of these parameters. The values of all these parameters indicate that we are observing methyl group rotation. We note that the slow-motion limit $(\omega \tau>>$ 1) for this motion would involve temperatures well below $80 \mathrm{~K}$ (the lowest temperature reported here) in which case quantum mechanical tunelling ${ }^{31}$ would play a role. The above expressions for $R_{\mathrm{S}}$ must then be modified but $E_{\mathrm{NMR}}=5 \pm 1 \mathrm{~kJ} \mathrm{~mol}^{-1}$ would not be affected. In addition to the $R_{\mathrm{S}}$ values, values of $R^{*}$ from the stretched exponential are shown in Fig. 5 solely to indicate that $R_{\mathrm{S}}$ is significantly larger than $R^{*}$. This difference correlates with the value of $\beta=0.87 \pm 0.02$ in Fig. 4 .

A modified version of the above discussion can be applied to the (almost exponential) ${ }^{1} \mathrm{H}$ spin-lattice relaxation resulting from the motion of the $t$-butyl group and its constituent methyl groups. The details are somewhat complicated and are presented elsewhere. ${ }^{33,38,39}$ In the most general case there are four $\tau$ values (and, therefore, four activation energies $E_{\mathrm{NMR}}$ ); one for the $t$ butyl group and one for each of the three methyl groups. The expressions for $R$ are tedious to generate but not conceptually difficult to understand: $R=\sum_{\mathrm{i}} C_{\mathrm{i}}\left[J\left(\omega, \tau_{\mathrm{i}}\right)+4 J\left(2 \omega, \tau_{\mathrm{i}}\right)\right]$. In this case, the $C_{\mathrm{i}}$ absorb the $n_{\mathrm{i}} / N$, the $A_{\text {intra }}$ and some of the $A_{\text {inter. }}$. They account for both the $3 \times 6=18$ intraCH${ }_{3}{ }^{1} \mathrm{H}-{ }^{1} \mathrm{H}$ interactions within the three $\mathrm{CH}_{3}$ groups and the $3 \times 3 \times 3=27$ interCH $\mathrm{X}_{3}$, intra- $t$ butyl, ${ }^{1} \mathrm{H}-{ }^{1} \mathrm{H}$ interactions. There are seven terms in the sum; one for the $t$-butyl group, one for each of the three methyl groups, and one each for the superimposed rotation of the $t$-butyl group and the three methyl groups. There are a variety of possibilities for the relative values of the four $\tau$ values. The simplest model that fits the ${ }^{1} \mathrm{H}$ spin-lattice relaxation rate data at high temperature reported here (which encompasses both the $\omega \tau<<1$ and $\omega \tau>>1$ regimes) is one where the $t$-butyl group and one methyl group rotate with $\tau_{\mathrm{b}}$ and the other two methyl groups rotate with $\tau_{\mathrm{c}}$. By "simplest" fit we mean the fit having the smallest number of adjustable parameters. (The choice of subscripts 
' $\mathrm{b}$ ' and ' $\mathrm{c}$ ' is to be consistent with previous publications where the model is laid out in detail. ${ }^{38,39}$ ) Thus $\tau_{\mathrm{b}}=\tau_{0 \mathrm{~b}}\left[\exp \left(E_{\mathrm{NMRb}} / k T\right)\right]$ and $\tau_{\mathrm{c}}=\tau_{0 \mathrm{c}}\left[\exp \left(E_{\mathrm{NMRc}} / k T\right)\right]$. There are terms in the expression of the relaxation rate in $\tau_{\mathrm{b}}, \tau_{\mathrm{c}}, \tau_{\mathrm{bb}}\left(\tau_{\mathrm{bb}}{ }^{-1}=\tau_{\mathrm{b}}{ }^{-1}+\tau_{\mathrm{b}}{ }^{-1}=2 \tau_{\mathrm{b}}{ }^{-1}\right)$, and $\tau_{\mathrm{bc}}\left(\tau_{\mathrm{bc}}{ }^{-1}=\tau_{\mathrm{b}}{ }^{-1}+\tau_{\mathrm{c}}{ }^{-1}\right)$ where $\tau_{\mathrm{bb}}$ is the correlation time characterizing the superimposed motion of one methyl group and the $t$-butyl group and $\tau_{\mathrm{bc}}$ is the correlation time characterizing the superimposed motion of the other two methyl groups and the $t$-butyl group. The strengths $C_{\mathrm{i}}$ (which include the appropriate values of $n_{\mathrm{i}} / N, A_{\text {intra }}$ and some of the $A_{\text {inter }}$ ) can all be related to $A_{\text {intra }}$ as well as other numerical factors. ${ }^{33,38,39}$

The best fit of the high temperature $R$ versus $T$ data in Fig. 5 is found for all the constants $C_{\mathrm{i}}$ set to their calculated values. ${ }^{38,39}$ The remaining fitted parameters are $E_{\mathrm{NMRb}}=18.6 \mathrm{~kJ} \mathrm{~mole}^{-1}$, $E_{\mathrm{NMRc}}=14.1 \mathrm{~kJ} \mathrm{~mole}^{-1}, \tau_{0 \mathrm{~b}} / \tau_{\mathrm{HOb}}=0.2$, and $\tau_{0 \mathrm{c}} / \tau_{\mathrm{HOc}}=2$. The uncertainties on each $E_{\mathrm{NMR}}$ are approximately $\pm 7 \%$ if the other three parameters are frozen at the above values but if all four of these parameters are allowed to vary the uncertainty is $\pm 15 \%$. The uncertainties in the $\tau_{0} / \tau_{\mathrm{HO}}$ values are approximately $\pm 50 \%$ (since they are multiplied by an exponential factor which, itself, contains a parameter with an uncertainty). The success of this fit, particularly the fact that the $C_{\mathrm{i}}$ values take on their a priori computed values ${ }^{38,39}$ means that the silyloxy group and its constituent silyl group are not moving on the NMR time scale. Probably all three $t$-butyl methyl groups have slightly different NMR activation energies and if that were allowed in the fit of the data, the parameter space would be much larger and the assignments would not be unique. So, we emphasize that the parameters assigned here represent the smallest parameter space that will fit the data.

Finally, we note that the temperature range where the low-temperature Si methyl group rotation and the high-temperature $t$-butyl+methyl group rotation are both contributing to the observed relaxation was not used in the fits for the two regions $(98-130 \mathrm{~K}$ at $8.50 \mathrm{MHz}, 110-140 \mathrm{~K}$ at $22.5 \mathrm{MHz}$, and $120-150 \mathrm{~K}$ at $53.0 \mathrm{MHz}$ in Fig. 5). That the sums of the two contributions fit the data in this intermediate temperature region is reassuring and also suggests that the motions can be treated independently. To put this another way, at low temperatures the $t$-butyl group and its constituent methyl groups are rotating so slowly as to appear static (on the NMR time scale) and only the rotation of the Si methyl groups is observed. At the other extreme, at high temperatures, the Si methyl groups are rotating so fast (on the NMR time scale) as to not contribute to the relaxation and only $t$-butyl+constituent methyl group rotation is observed. 


\section{Conclusions}

In van der Waals organic solids, the atoms in the molecules are held together by strong covalent interactions and the molecules are held together in the solid by much weaker van der Waals interactions. ${ }^{40}$ The molecules generally keep their 'identity' in the solid state, even though there may be some conformational changes. The feature of interest in the particular compound reported here (2-t-butyldimethylsilyloxy-6-bromonaphthalene) is that the silyloxy group provides two "lone" methyl groups bonded to a Si atom and three methyl groups in a $t$-butyl group (See Fig. 1). All of this is firmly "anchored" in the solid state by the silyloxy backbone attached to an aromatic ring system. The fit of the solid state ${ }^{1} \mathrm{H}$ spin-lattice relaxation rate data shows that the larger asymmetric silyloxy group and its constituent silyl group may rotationally librate slightly but these motions will involve small angular oscillations and will be at a much higher frequency than the NMR frequency. As such, these motions do not contribute (directly) to the ${ }^{1} \mathrm{H}$ spin-lattice relaxation process. They add a very fast (and small angular) time dependence to the rotational axes for the various methyl and $t$-butyl groups in addition to the spatial randomization of these rotation axes as a consequence of the polycrystalline nature of the sample. Indeed, we know, from previous experience that in the solid state, even for small asymmetric groups like methoxy, ethyl, and isopropyl groups, rotation is completely quenched. ${ }^{5,41-43}$ This is not the case for a threefold symmetric $t$-butyl group which does reorient on the NMR time scale.

The most important parameter determined by the solid state NMR relaxation experiments is the NMR activation energy $E_{\mathrm{NMR}}$. (Other parameters are important but mainly to both allow us to say we are indeed looking at methyl and $t$-butyl group rotation and to allow us to distinguish between the methyl groups bonded to the Si atom and those in the $t$-butyl group.) $E_{\mathrm{NMR}}$ can be related to a barrier for methyl group rotation ${ }^{44-48}$ and it is generally between 0 and $20 \%$ smaller than the barrier. ${ }^{46,47}$ As such, $E_{\mathrm{NMR}}$, which is measured to within $\pm 10 \%$ or so, can be a stand in for the rotational barrier. These barriers can be computed by electronic structure calculations in clusters of molecules (based on the X-ray structures) and the agreement between the computed barriers and the measured values of $E_{\mathrm{NMR}}$ in systems similar to (but somewhat less complicated than) that studied here is very good. ${ }^{2,3,5,6,42-43}$ Electronic structure calculations in systems involving $t$-butyl groups are very complicated ${ }^{2}$ because of the superimposed methyl and $t$-butyl group rotation. However, there are several additional possible motions in the silyloxy group in 2-tbutyldimethylsilyloxy-6-bromonaphthalene so calculations in clusters of molecules of this 
compound will be very challenging. Silyloxy and silyl group small angle, high frequency librations will not affect the NMR solid state relaxation but they will likely slightly reduce the methyl and $t$-butyl group barriers from values computed assuming that the silyloxy and silyl groups are rigid.

We are interested in rotation on a time scale 'seen' by nuclear magnetic resonance (NMR) ${ }^{1} \mathrm{H}$ relaxation experiments. In the solid state, the Si methyl groups in the system studied here rotate about their fixed axes (that is, fixed on the NMR time scale) with NMR activation energies of $E_{\mathrm{NMR}}=5 \pm 1 \mathrm{~kJ} \mathrm{~mol}^{-1}\left(1.0 \pm 0.2 \mathrm{kcal} \mathrm{mol}^{-1}\right)$. This is pretty much the lower limit on $E_{\mathrm{NMR}}$ that can be studied by the solid state NMR relaxation technique; we only observe these methyl groups $(>80 \mathrm{~K}$ ) on a time scale where methyl group rotation is faster than the inverse NMR frequency. These rotors are undoubtedly undergoing quantum mechanical tunelling ${ }^{31}$ at lower temperatures. The $t$-butyl group and its three component methyl groups are all rotating in a superimposed fashion on the NMR time scale and we see the entire dynamical regime; from rotating much more slowly than the inverse NMR frequency to rotating much faster than the inverse NMR frequency. The simplest fit suggests that the $t$-butyl group and one of its methyl groups is rotating with an NMR activation energy of $19 \mathrm{~kJ} \mathrm{~mole}^{-1}\left(4.5 \mathrm{kcal} \mathrm{mol}^{-1}\right)$ and the other two methyl groups are rotating with an NMR activation energy of $14 \mathrm{~kJ} \mathrm{~mole}^{-1}\left(3.3 \mathrm{kcal} \mathrm{mol}^{-1}\right)$. The NMR relaxation technique has its limitations and these NMR activation energies should be taken as a guide. Opening up the parameter space for the fits would suggest that the $t$-butyl group has a barrier of $17-21 \mathrm{~kJ}^{\text {mole }}{ }^{-1}$ (4.1-5.0 $\left.\mathrm{kcal} \mathrm{mol}^{-1}\right)$, that one of its methyl groups has a barrier of $16-21 \mathrm{~kJ} \mathrm{~mole}^{-1}(3.8-5.0 \mathrm{kcal}$ $\left.\mathrm{mol}^{-1}\right)$ and that the other two methyl groups have barriers in the $12-15 \mathrm{~kJ} \mathrm{~mole}^{-1}\left(2.9-3.6 \mathrm{kcal} \mathrm{mol}^{-}\right.$ ${ }^{1}$ ) range. The intramolecular and intermolecular interactions in this system are complicated and no doubt these barriers are all slightly different. These barriers are similar to those found in similar compounds with $t$-butyl groups. ${ }^{2,38,39,49-54}$

1 Kimmich, R. NMR Tomography, Diffusometry, Relaxometry (Springer-Verlag: Berlin, 1997).

2 X. Wang, F. B. Mallory, C. W. Mallory, H. R. Odhner and P. A. Beckmann, J. Chem. Phys., 2014, 140, 194304, 1-15.

3 P. A. Beckmann, K. G. Conn, C. W. Mallory, F. B. Mallory, A. L. Rheingold, L. Rotkina and X. Wang, J. Chem. Phys., 2013, 139, 204501, 1-12.

4 R. Tilley, Crystals and Crystal Structures (Wiley, Chichester, UK, 2006). 
5 P. A. Beckmann, C. W. Mallory, F. B. Mallory, A. L. Rheingold and X. Wang, ChemPhysChem, 2015, 16, 1509-1519.

6 X. Wang, P. A. Beckmann, C. W. Mallory, A. L. Rheingold, A. G. DiPasquale, P. Carroll and F. B. Mallory, J. Org. Chem., 2011, 76, 5170-5176.

7 R. Kohlrausch, Ann. Phys. Chem. (Poggendorff), 1854, 91, 179-214.

8 P. A. Beckmann, Sol. State Nuc. Mag. Resonan., 2015, in press, doi:10.1016/j.ssnmr.2015.07.001

9 P. A. Beckmann and E. Schneider, J. Chem. Phys., 2012, 136, 054508, 1-9.

10 R. Kahlau, D. Kruk, Th. Blochowicz, V. N. Novikov and E. A. Rossler, J. Phys. C: Solid State Phys., 2010, 22, 365101, 1-10.

11 T. C. Dotson, J. Budzien, J. D. McCoy and D. B. Adolf, J. Chem. Phys., 2009, 130, 024903, 19.

12 M. Berberan-Santos, E. N. Bodunov and B. Valeur, Ann. Phys. (Berlin), 2008, 17, 460-461.

13 D. C. Johnston, Phys. Rev. B , 2006, 74, 184430, 1-7.

14 R. G. Palmer, D. L. Stein, E. Abrahams and P. W. Anderson, Phys. Rev. Lett., 1984, 53, 958961.

15 J. R. Macdonald and J. C. Philips, J. Chem. Phys., 2005, 122, 074510, 1-8.

16 M. N. Berberan-Santos, E. N. Bodunov and B. Valeur, Chem. Phys., 2005, 315, 171-182.

17 R. L. Hilt and P. S. Hubbard, Phys. Rev., 1964, 134, A392-A398.

18 L. K. Runnels, Phys. Rev., 1964, 134, A28-A36.

19 M. F. Baud and P. S. Hubbard, Phys. Rev., 1968, 170, 384-390.

20 A. Kumar and C. S. Johnson, Jr., J. Chem. Phys., 1974, 60, 137-146.

21 J. D. Cutnell and W. Venable, J. Chem. Phys., 1974, 60, 3795-3801.

22 L. J. Burnett and B. H Muller, Chem. Phys. Lett., 1973, 18, 553-556.

23 S. Clough and A. Heidemann, J. Phys. C: Solid State Phys., 1980, 13, 3585-3589.

24 S. Clough, A. Heidemann, A. J. Horsewill, J. D. Lewis and M. N. J. Paley, J. Phys. C: Solid State Phys., 1981, 14, L525-L529.

25 S. Clough and P. J. McDonald, J. Phys. C: Solid State Phys., 1982, 15, L1039-L1042.

26 S. Clough, P. J. McDonald and F. O. Zelaya, J. Phys. C: Solid State Phys., 1984, 17, 44134420.

27 D. Cavagnat, S. Clough and F. O. Zelaya, J. Phys. C: Solid State Phys., 1985, 18, 6457-6462.

28 S. Clough, Physica, 1986, 136B, 145-149.

29 M. J. Barlow, S. Clough, A. J. Horsewill and M. A. Mohammed, Solid State Nuc. Mag. Resonan., 1992, 1, 197-204.

30 S. Clough, Solid State Nuc. Mag. Resonan., 1997, 9, 49-53. 
31 A. J. Horsewill, Prog. Nuc. Mag. Resonan. Spectros., 1999, 35, 359-389.

32 E. O. Stejskal and H. S. Gutowsky, J. Chem. Phys., 1958, 28, 388-396. See a correction in the footnote on page 973 of ref. 33.

33 M. B. Dunn and C. A. McDowell, Molec. Phys., 1972, 24, 969-978.

34 K. G. Conn, P. A. Beckmann, C. W. Mallory and F. B. Mallory, J. Chem. Phys., 1987, 87, $20-$ 27.

35 J. S. Waugh and É. I. Fedin, Soviet Physics - Solid State, 1963, 4, 1633-1636.

36 R. Ferrando, R. Spadacini, G. E. Tommei and V. I. Mel'nikov, Phys. Rev. E, 1995, 51, R1645R1648.

37 N. L. Owen, in Internal Rotation in Molecules, edited by W. J. Orville-Thomas (Wiley, New York, 1974).

38 P. A. Beckmann, A. I. Hill, E. B. Kohler and H Yu, Phys. Rev. B, 1988, 38, 11098-11111.

39 P. A. Beckmann, C. A. Buser, K. Gullifer, F. B. Mallory, C. W. Mallory, G. M. Rossi and A. L. Rheingold, J. Chem. Phys., 2003, 118, 11129-11138.

40 IUPAC, Org. Chem. Div., Pure \& Applied Chem., 1994, 66, 1077, see p 1175. Also available at (http://goldbook.iupac.org/V06597.html).

41 X. Wang, L. Rotkina, H. Su and P. A. Beckmann, ChemPhysChem, 2012, 13, 2082-2089.

42 D. P. Fahey, W. G. Dougherty Jr., W. S. Kassel, X. Wang and P. A. Beckmann, J. Phys. Chem. A, 2012, 116, 11946-11956.

43 X. Wang, A. L. Rheingold, A. G. DiPasquale, F. B. Mallory, C. W. Mallory and P. A. Beckmann, J. Chem. Phys., 2008, 128, 124502, 1-3.

44 A. Detken, P. Focke, H. Zimmermann, U. Haeberlen, Z. Olejniczak and Z. T. Lalowicz, Z. Naturforch., 1995, 50a, 95-116.

45 T. K. Jahnke, W. Müller-Warmuth and M. Bennati, Solid State Nucl. Mag. Resonan., 1995, 4, 153-161.

46 O. Edholm and C. Blomberg, Chem. Phys., 1981, 56, 9-14.

47 J. Kowaleski and T. Liljefors, Chem. Phys. Lett., 1979, 64, 170-174.

48 E. K. van Putte, J. Mag. Resonan., 1970, 2, 216-224.

49 L. C. Popa, A. L. Rheingold and P. A. Beckmann, Solid State Nuc. Mag. Resonan., 2010, 38, 31-35.

50 P. A. Beckmann, W. G. Dougherty Jr. and W. S. Kassel, Solid State Nuc. Mag. Resonan., 2009, 36, 86-91.

51 A. L. Rheingold, A. G. DiPasquale and P. A. Beckmann, Chem. Phys., 2008, 345, 116-118.

52 P. A. Beckmann, C. Paty, E. Allocco, M. Herd, C. Kuranz and A. L. Rheingold, J. Chem. Phys., 2004, 120, 5309-5314.

53 P. A. Beckmann, K. S. Burbank, M. M. W. Lau, J. N. Ree and T. L. Weber, Chem. Phys., 2003, 290, 241-250. 
54 P. A. Beckmann, K. S. Burbank, K. M. Clemo, E. N. Slonaker, K. Averill, C. Dybowski, J. S. Figueroa, A. Glatfelter, S. Koch, L. M. Liable-Sands and A. L. Rheingold, J. Chem. Phys., 2000, 113, 1958-1965.

Table 1. Crystallographic Data for 2-t-butyldimethylsilyloxy-6-bromonaphthalene

\begin{tabular}{|l|l|}
\hline Empirical Formula & $\mathrm{C}_{16} \mathrm{H}_{21} \mathrm{BrOSi}$ \\
\hline CCDC deposit number & 1420396 \\
\hline Formula Weight & 337.33 \\
\hline Temp $(\mathrm{K})$ & 100 \\
\hline Wavelength $(\AA)$ & 0.71073 \\
\hline Crystal System & monoclinic \\
\hline Space Group & $P 2_{1}$ \\
\hline$a(\AA)$ & $7.0513(3)$ \\
\hline$b(\AA)$ & $7.6501(2)$ \\
\hline$c(\AA)$ & $15.5178(7)$ \\
\hline$\beta\left(^{\circ}\right)$ & $101.3670(10)$ \\
\hline$V\left(\AA^{3}\right)$ & $820.66(6)$ \\
\hline$Z$ & 2 \\
\hline$R$ flns (collect/indepnt) & $4924 / 2742$ \\
\hline$R 1, w R 2(2 \sigma(\mathrm{I}))$ & $1.86,4.24$ \\
\hline Flack parameter & $0.017(6)$ \\
\hline GOF & 0.874 \\
\hline
\end{tabular}




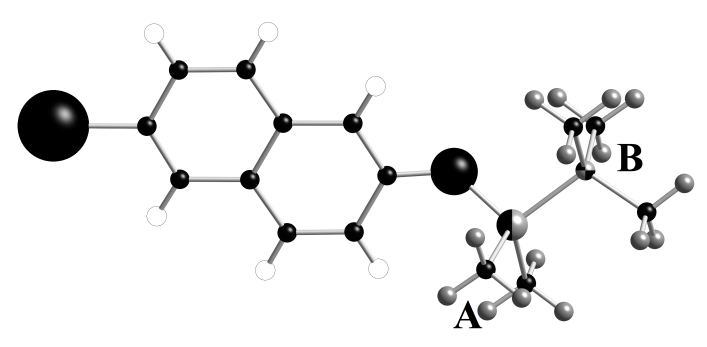

(a)

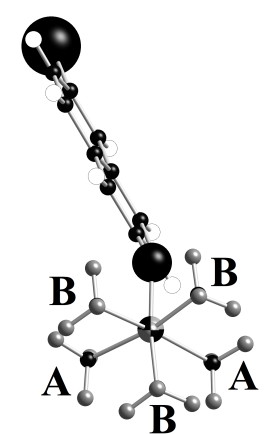

(b)

Fig. 1. Two views of a molecule of 2-t-butyldimethylsilyloxy-6-bromonaphthalene in the crystal. The asymmetric unit is a single molecule so all molecules are equivalent. The large black sphere is the $\mathrm{Br}$ atom, the medium-sized black sphere is the $\mathrm{O}$ atom, the two-tone sphere bonded to the $\mathrm{O}$ atom is the $\mathrm{Si}$ atom, the small black beach ball sphere bonded to the Si atom is the $t$-butyl group quaternary $\mathrm{C}$ atom $(\mathrm{Ct})$, the small black spheres are the other silyloxy group and ring $\mathrm{C}$ atoms, the small hollow white spheres are ring $\mathrm{H}$ atoms, and the small grey spheres are the silyloxy group $\mathrm{H}$ atoms. Methyl groups attached to the $\mathrm{Si}$ atom are labeled $\mathrm{A}$ and methyl groups in the $t$-butyl group are labeled B. The projection in (a) is perpendicular to the plane of the aromatic rings and the projection in (b) has the $\mathrm{Si}-\mathrm{Ct}$ bond perpendicular to the page. 

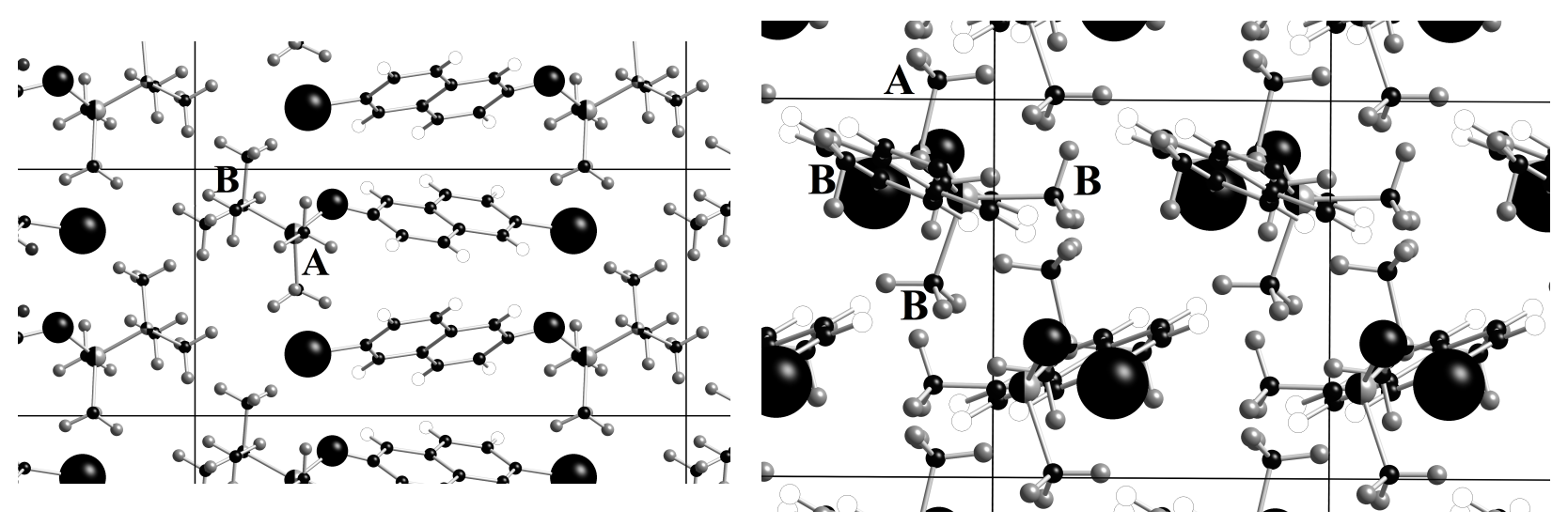

(a)

(b)

Fig. 2. The crystal structure of 2-t-butyldimethylsilyloxy-6-bromonaphthalene in the (a) 100 and (b) 101 planes. All molecules are equivalent and two projections of the molecule are shown in Figs. 1a and $\mathrm{b}$. The symbols that identify the various atoms are explained in the caption to Fig. 1. Methyl groups attached to the $\mathrm{Si}$ atom are labeled A and methyl groups in the $t$-butyl group are labeled B. In (b), only one $\mathrm{H}$ atom on one of the methyl groups in the $t$-butyl group is visible (far left below the B) and one of the methyl groups bonded to the Si atom is well hidden into the page. 


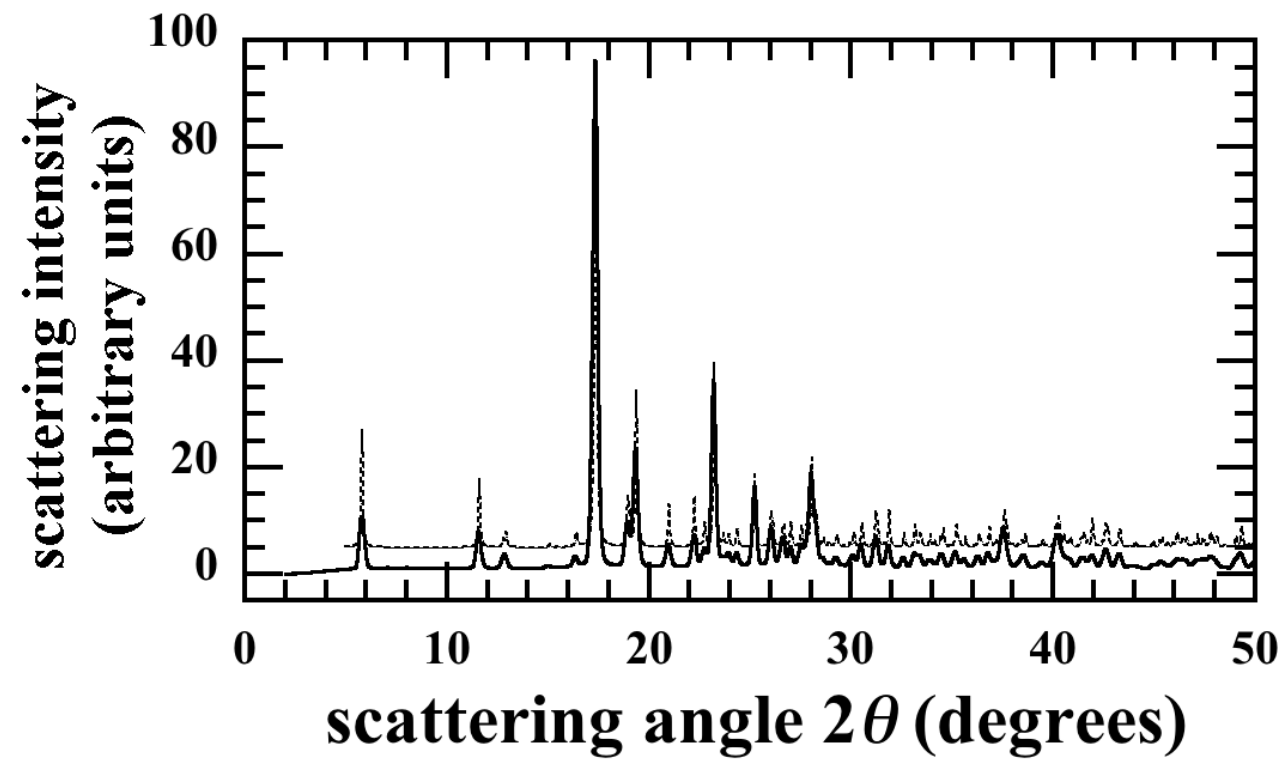

Fig. 3. A comparison of the single crystal and powder X-ray diffractograms. The solid line shows the powder diffractogram and the dashed line (offset vertically upwards for clarity) shows the powder diffractogram computed from the single crystal X-ray data. 


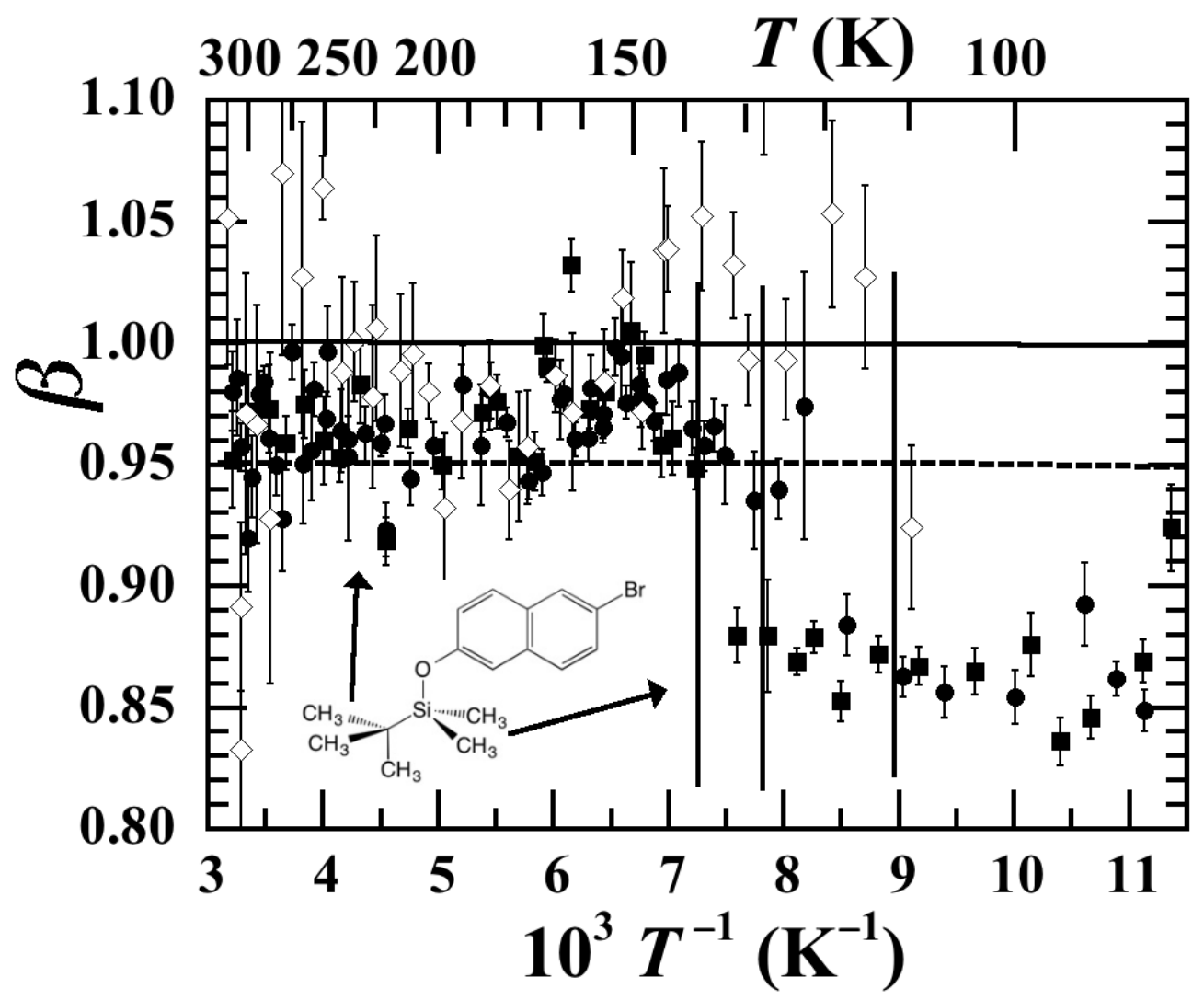

Fig. 4. The stretching parameter $\beta$ versus inverse temperature $T^{-1}$ at $53.0(\boldsymbol{\square}), 22.5(\mathbf{O})$, and 8.50 $\mathrm{MHz}(\diamond)$. The solid horizontal line at $\beta=1$ indicates exponential relaxation. When $\beta>0.95$ (dashed horizontal line) $R$ (the unique rate in a single exponential) is indistinguishable from $R^{*}$ (the characteristic rate in a stretched exponential) within experimental uncertainty and the relaxation is deemed exponential. The vertical lines (left for $53.0 \mathrm{MHz}$, center for $22.5 \mathrm{MHz}$, and right for $8.50 \mathrm{MHz}$ ) indicate the transitions from exponential relaxation at high temperatures characterized by $R$ (Fig. 5) to nonexponential relaxation at low temperatures characterized by $R_{\mathrm{S}}$, $R^{*}$ (Fig. 5) and $\beta$. The arrows from the molecule indicate the intramolecular groups responsible for relaxation in the two temperature regions. 


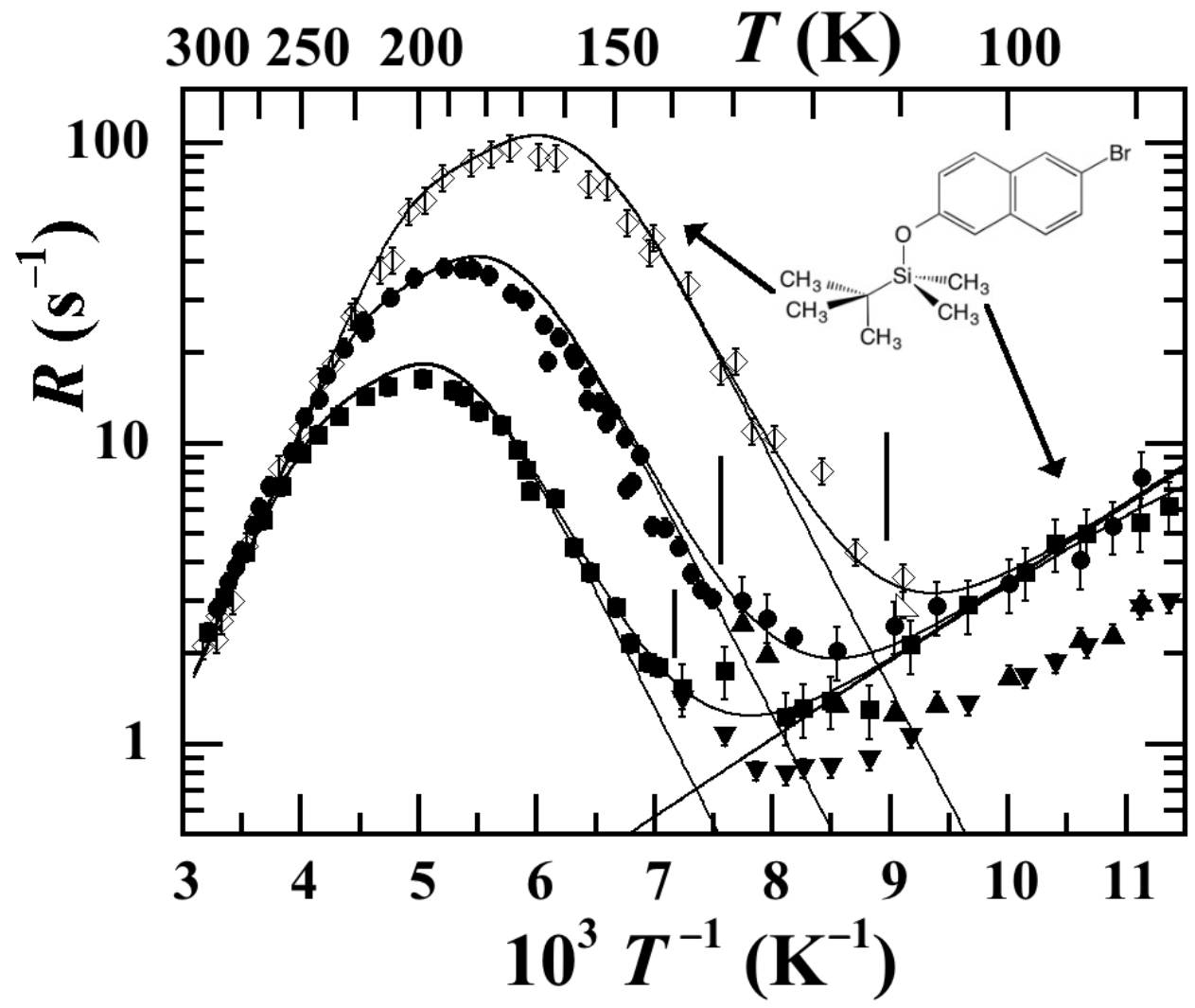

Fig. 5. The ${ }^{1} \mathrm{H}$ spin-lattice relaxation rates $R(\boldsymbol{\square}, \boldsymbol{\bigcirc}, \diamond), R_{\mathrm{S}}(\boldsymbol{\square}, \boldsymbol{\bigcirc}, \diamond)$, and $R^{*}(\boldsymbol{\nabla}, \boldsymbol{\Delta}, \triangleleft)$ versus inverse temperature $T^{-1}$ at $53.0(\boldsymbol{\square}, \boldsymbol{\nabla}), 22.5(\boldsymbol{\bullet}, \boldsymbol{\Delta})$, and $8.50 \mathrm{MHz}(\diamond, \triangleleft)$. The vertical lines (left for $53.0 \mathrm{MHz}$, center for $22.5 \mathrm{MHz}$, and right for $8.50 \mathrm{MHz}$ ) indicate the transitions from exponential relaxation at high temperatures characterized by $R$ to nonexponential relaxation at low temperatures characterized by $R^{*}$ and $R_{\mathrm{S}}$. The arrows from the molecule indicate the intramolecular groups responsible for relaxation in the two temperature regions. 